\title{
A Model of Fatigue Crack Propagation in Metals
}

\section{T. Yu. Yakovleva and L. E. Matokhnyuk}

Pisarenko Institute of Problems of Strength, National Academy of Sciences of Ukraine, Kiev, Ukraine

A model of the formation and evolution of a local plastic deformation zone at the crack tip is proposed based on the analysis of the main physical processes taking place in a metallic material under the action of cyclic loads. An equation of fatigue crack growth rate curves, which explicitly accounts for the loading frequency, was derived. The equation applies to the whole range of crack lengths from short cracks to macroscopic ones.

Keywords: local plastic deformation, surface energy, fatigue strength, fatiguecrack growth resistance, loading frequency.

Introduction. Fracture of a material and structural element under external thermomechanical loading is a two-stage process. The first stage involves damage accumulation in the material and comes to an end when the parameters of the local plastic deformation zone reach their critical values, which corresponds to the beginning of the formation of one or several cracks. The second stage is characterized by the crack propagation up to a complete body failure. Nowadays, there are attempts to describe the entire process of fatigue fracture from a single perspective, with the leading role given to the process zones which are formed both during the first (incubation) period and at the crack growth stage [1-6]. The growing fatigue crack is regarded as a sharp notch and its growth is modeled as repeated crack initiation events which follow the same laws as those governing the initiation of the primary crack.

The major characteristics of the loading conditions include the frequency of the acting load. The few models considering the frequency either contain it in an implicit form [7], or cover only one or several materials [8-10], or are difficult to apply in practice [11].

Based on the above, what seems topical to us is the creation of unified models covering the widest possible range of factors that influence the fatigue fracture process, relying on the analysis of physical processes that take place in a metallic material, and having a sufficiently simple, easy-to-use mathematical form.

Physical Foundations of the Fracture Model. Earlier we analyzed experimental data, both our own and those from literature, on the processes of fatigue damage accumulation and fatigue crack propagation in metallic materials using such techniques as optical, transmission electron, and scanning electron microscopies combined with a quantitative data processing and the determination of the residual electrical resistivity and internal friction. This made it possible to establish basic general laws for the evolution of the material structure and variation of the fractographic characteristics under cyclic loading. Detailed results of these investigations are given in [12]. Here we outline only the key issues. 
The main distinction of the structural changes occurring during the period of fatigue damage accumulation is their local nature. Progressive microplastic deformation is observed in a limited number of microvolumes, along with a much larger number of non-progressive zones of structural changes appearing in the material. The microvolumes of the maximum structural changes have a dislocation structure different from that of the original material and are surrounded by a zone of mild changes, which is similar to a transition zone to the rest of the material, being almost in the same state as before loading. The extent of the structural changes in different microvolumes depends significantly on the sensitivity of their crystalline structure to the direction of the loading axis, as well as on the local stress concentration, nonuniformity in terms of impurities and alloying elements, and closeness to the free material surface.

The increase in the degree of localization of the material restructuring with rising duration of loading was revealed by means of direct strain measurements [13], Barkhausen noise analysis [14], and Fourier methods [15, 16]. Fracture occurs when a certain critical state of the structure is attained in the sites of localization of deformation.

As the loading frequency increases, the number of defects accumulated over a loading cycle, as well as the size of the zones with marked structural changes, decreases. The impeded realization of the plastic deformation micromechanisms because of the shorter cycle duration under high-frequency loading is made up for by the activation of the micromechanisms that are less energetically feasible under low frequencies. Still, the basic laws of damage accumulation described above hold true.

The presence of the static component under cyclic loading and an increase in the stress ratio $R$ result in smaller degrees of localization of deformation, which shows up in a slight reduction of the material volume that underwent no structural changes [12].

The dislocation structure of the plastic deformation zone at the tip of the main crack in the near-threshold range of the stress intensity factor $\Delta K$ is the next logical stage in the evolution of the structure formed during the fatigue damage accumulation in the original material. The material exhibits quantitative rather than qualitative differences between the dislocation structure formed in the fracture zone and the local microzones in the original material most heavily deformed during the incubation period of the fatigue fracture. The plastic deformation process in the fracture zone engulfs almost entire microvolume located in the area affected by the crack tip.

Comparison of the fracture surface and the corresponding dislocation structure suggests that the length of the preparatory period preceding the material fracture has a significant effect on the crack growth rate both prior to the initiation and during the propagation of the primary crack. Fractographic investigations [17] and direct observations of the fatigue-crack growth process [18] show the possibility of using the "microcrack at the notch-tip" model to represent the process of the macrocrack extension increment. For this model, the tip of a macrocrack at its blunting stage plays the role of the notch.

At the stage of propagation of the main crack, as well as during the incubation period, the growth of the loading frequency is accompanied by the 
reduction of the element size in the substructure being formed. The band width in a band structure [19] and the depth of the plastic deformation zone [20] increase less intensively with $\Delta K$ as compared with their increase under low-frequency loading.

Analysis of the research findings summarized here allows us to draw the following conclusions.

1. Zones of progressive local plastic deformation (LPD zones) can be regarded as parts of some "quasi-phase," which differ in properties from the remaining material and, consequently, their specific surface energy depends on the coordinates.

2. The size of these zones depends on the local stress state, micromechanisms of plastic deformation, number of cycles, and loading frequency, and the local fracture of the material occurs as a result of the loss of its capacity for further plastic deformation.

3. The evolution of the LPD zones in a given material during the incubation period and the active period of fatigue fracture is governed by common laws.

4. The fatigue-crack growth rate is mainly determined by the duration of the preparatory period in the restructuring of the LPD zone material, and this duration, in turn, depends on the material properties and levels of nominal stresses.

5. The loading frequency has a similar impact on the integral macroscopic and local (structural and fractographic) characteristics of the fatigue fracture.

The Model of Material Fracture under Cyclic Loading. Consider a model for the behavior of a metallic material subjected to a cyclic load of arbitrary magnitude with the frequency $f$ taking into account the above research findings on the related physical processes. To this end, we make the following preliminary assumptions:

(i) the LPD zone material is a continuous medium whose physical-mechanical properties, including the specific surface energy, are functions of the coordinates and time;

(ii) the current mean radius of this zone depends linearly on the mean rate of the microplastic deformation process and time;

(iii) the exact nature and mechanisms of the structure evolution are reflected in changes in the specific surface energy.

Starting from the above assumptions, we first analyzed the equilibrium conditions for the system $L P D$ zone - remaining material volume taking into account the presence of an interfacial area and then the energy balance within the LPD zone.

In the case studied, under the ultimate equilibrium conditions the component inducing macroscopically elastic deformation should be considered together with three additional components. These are the dissipative force [21], which is related to the irreversible energy dissipation within a cycle and depends on the cycle characteristics, and the components dependent on the resulting surface curvature within the LPD zone and the magnitudes of the interaction forces acting along the interface between the main volume and the LPD zone. The latter components are linked to the size of the LPD zone and the value of the specific surface energy $\alpha$ accumulated within this zone after $N$ loading cycles, i.e., they depend on the 
duration of loading. The stresses $\sigma$ in the case under study can also be presented as a sum of the $\sigma_{e}$ component inducing macroelastic deformation and the $\sigma_{m d}$ component introduced to account for the energy dissipation and the presence of the interface between the LPD zone and the remaining material. We present $\sigma_{m d}$ as a sum of three components, two of them being dependent on the duration of loading and the third one on the cycle characteristics:

$$
\sigma=\sigma_{e}+\sigma_{m d}
$$

where

$$
\sigma_{m d}=\sigma_{m d 1}+\sigma_{m d 2}+\sigma_{m d 3}
$$

When analyzing variation of the internal energy within the LPD zone with time $t$, we also considered two conditional components: the first one, linked to the size of the plastic deformation zone, and the second one, linked to the evolution of the structural morphology within this zone and, therefore, variation of the specific surface energy $\alpha$. In the same manner, we also divide the work of external forces into two conditional components. The first one, being the work of the elastic deformation within one loading cycle, is equal to zero. The second component is the work done over the time $t$ to bring the LPD microvolume to a nearly spherical shape with a characteristic linear dimension, e.g., mean radius. The LPD zone expansion continues as long as the decrease in its energy due to the stress relaxation caused by the structural evolution is greater than the increase in this energy caused by the growth of the geometric dimension and the specific surface energy. The critical moment is when the sum of changes in the energy equals zero, which corresponds to certain critical values of the zone size and specific surface energy. A further decrease in the energy is only possible through fracture in the zone, i.e., the appearance of a primary microcrack. The condition for attaining the maximum of the function of several variables is the equality to zero of the partial derivatives, and the local fracture criteria are the critical values of the LPD zone radius and specific surface energy. As a result, we arrive at a relationship that is a constitutive equation for the material in the state of instability accounting for the cyclic loading rate and stress ratio:

$$
\sigma_{a}=\sigma_{e}+a_{\sigma} \sqrt{f}+b_{\sigma} \sqrt{\frac{1}{N_{F r}}}+c_{\sigma} \sqrt{\frac{f}{N_{F r}}},
$$

where

$$
\begin{gathered}
a_{\sigma}=k \sqrt{\frac{E_{\omega} \tan \varphi}{3 v_{0}} \frac{\partial \alpha}{\partial N}}, \quad b_{\sigma}=k \sqrt{\frac{E_{r}}{3 v_{0}} \frac{\partial \alpha}{\partial T}}, \quad c_{\sigma}=k \sqrt{\frac{E_{\omega} \tan \varphi}{3} \frac{\partial \alpha}{\partial v_{0}}}, \\
\sigma_{e}=k \sigma_{e /-1}, \quad k=1-k_{a / R} \frac{1+R}{2},
\end{gathered}
$$

$\varphi$ is the phase shift angle between the stress and strain, $E_{\omega}$ and $E_{r}$ represent the circumferential elastic modulus and relaxation modulus, respectively, [22], $k_{a / R}$ characterizes the material sensitivity to the static stress component, $\sigma_{e /-1}$ is the 
true elastic limit for fully-reversed cycles, and $v_{0}$ is the mean rate of microplastic deformation processes.

Further loading will lead to the onset of fracture. Reduced to its simplest form, this relationship is a fatigue curve equation with two coefficients for given stress-controlled loading conditions:

$$
\sigma_{a}=\sigma_{u e}+\frac{C_{f}}{\sqrt{N_{F r}}}, \quad \sigma_{u e}=\sigma_{e}+a_{\sigma} \sqrt{f}, \quad C_{f}=b_{\sigma}+c_{\sigma} \sqrt{f} .
$$

Dividing all the terms in Eqs. (2) and (3) by the value of the corresponding modulus, we obtain equations of similar form for the strain-controlled loading conditions.

Keeping in mind the above results, we consider the material state after the onset of the fatigue microcrack propagation under conditions of uniaxial tension.

The boundary of the LPD zone is itself an inner stress raiser and so, if we do not dwell on the specific micromechanisms depending on numerous factors, the most probable direction of the local fracture is from the boundary of the LPD zone into its inner volume at some angle $\beta$ to the loading axis in the plane of the maximum local tensile stresses (Fig. 1). If $2 l$ is the length of the initial microcrack, which corresponds to the first instantaneous event of local fracture, we arrive at the problem of a crack, which is modeled by a slant slot in the tensile stress field [23].

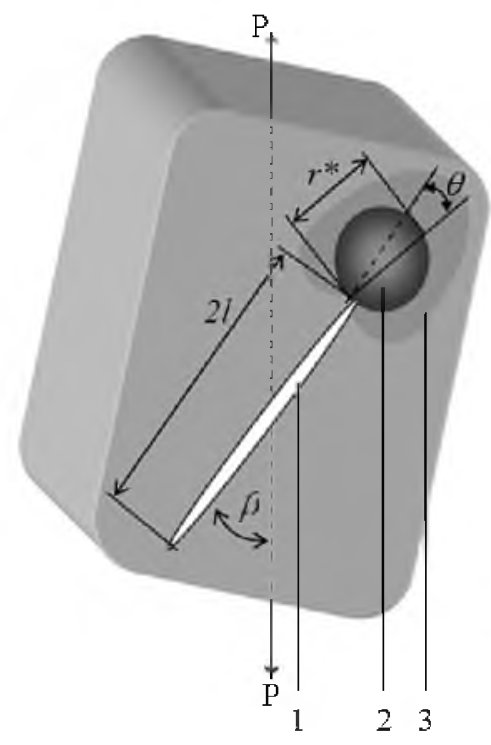

Fig. 1. Crack growth scheme: (I) primary crack; (2) secondary LPD zone; (3) transition zone.

Now consider the material state in the vicinity of the point $r, \theta$ (where $r, \theta$ is the local polar coordinate system in the crack cross-section), which belongs to a microzone exhibiting the above listed characteristics, namely, most intense structural changes, highest stresses, and their small gradient. We will call this microzone secondary LPD zone (Fig. 1), in contrast to the primary one formed 
during the incubation period. Then if $r \approx r^{*} / 2[r$ is the distance from the crack tip for which $\sigma_{a r i k}(r)=\sigma_{a r / i k \max }$ holds true], the local stresses $\sigma_{a r / i k}$ (and strains $\varepsilon_{a r / i k}$ ) within the microvolume in question weakly depend on $\Delta r$ [24]. If the value of the stress intensity factor is given, we can write the known expression for local stresses in the central point of the secondary LPD zone:

$$
\begin{gathered}
\sigma_{a r / i k}=\frac{\Delta K}{\sqrt{2 \pi r}} \Phi, \quad \Phi=F(r * / \rho) \Theta_{i k}(\theta) \Psi(\beta), \\
\Delta K=\sum_{n} \Delta K_{n}, \quad \Delta K_{n}=\sigma_{a 0 / i k} \sqrt{\pi l} Y, \quad \Theta_{i k}(\theta)=\sum_{n} \Theta_{i k / n}(\theta),
\end{gathered}
$$

where $i, k=x, y, z, n=\mathrm{I}$, II III characterizes the three basic modes of crack-tip opening displacement (mode I is the opening, mode II is the in-plane shear, and mode III is the out-of-plane shear), $\Theta_{i k}$ and $\Psi$ are functions of the angles $\theta$ and $\beta$, respectively, $\beta$ is the angle between the loading axis and the fracture propagation direction (Fig. 1), $Y$ is the correction function dependent on the ratio of $l$ to the specimen width, $F$ is the function dependent on the ratio $r^{*} / \rho$, where $\rho$ is the crack tip radius, and $\sigma_{a 0 / i k}$ is the amplitude of nominal stresses. Further, we will omit the indices $i$ and $k$ to avoid too lengthy designations of stresses.

Writing these stresses as a function of the number of cycles required to fracture the secondary zone,

$$
\sigma_{e r}+a_{\sigma r} \sqrt{f}+\left(b_{\sigma r}+c_{\sigma r} \sqrt{f}\right) \sqrt{\frac{1}{N}}=\frac{\Delta K \Phi}{\sqrt{2 \pi r}}
$$

we can present the function differential as a dependence of the crack growth rate on $\Delta K$ :

$$
\frac{d l}{d N}=\frac{2\left(\Delta K-\Delta K_{e}-a_{\Delta K} \sqrt{f}\right)^{2}}{\frac{\pi}{\Phi^{2}}\left(b_{\sigma r}+c_{\sigma r} \sqrt{f}\right)^{2}}, \quad \Delta K=k \Delta K_{-1},
$$

where

$$
k=1-k_{a /-1} \frac{1+R}{2}, \quad \Delta K_{e}=\frac{\sigma_{e r} \sqrt{2 \pi r}}{\Phi}, \quad a_{\Delta K}=\frac{\alpha_{\sigma r} \sqrt{2 \pi r}}{\Phi},
$$

or on the crack length:

$$
\frac{d l}{d N}=\frac{2\left[\sigma_{a 0} \sqrt{\pi l} Y-\frac{\sqrt{2 \pi r}}{\Phi}\left(\sigma_{e r}+a_{\sigma r} \sqrt{f}\right)\right]^{2}}{\frac{\pi}{\Phi^{2}}\left(b_{\sigma r}+c_{\sigma r} \sqrt{f}\right)^{2}}
$$


Here, the parameters $\sigma_{e r}, a_{\sigma r}, b_{\sigma r}$, and $c_{\sigma r}$ have the same physical and mathematical meaning as in Eq. (2), but refer to the microzone of the maximum structural changes in the vicinity of the point $r, \theta$ at the crack tip, $k_{a /-1}$ has the same meaning as in Eq. (2) and refers to the amplitude of external load, and $\Delta K_{-1}$ is the stress intensity factor for fully-reversed loading with a given crack growth rate.

The resulting equations can be reduced to a modified Paris law

$$
\frac{d l}{d N}=A_{f}\left(\Delta K-\Delta K_{u e}\right)^{2}
$$

i.e., expressed as a function of $\Delta K$. Here, the expressions for the coefficient and the addend account for the loading frequency and the expression for $\Delta K$ incorporates the stress ratio.

Similarly, we can simplify the relation between the crack growth rate and crack length:

$$
\begin{gathered}
\frac{d l}{d N}=A_{f}\left(B_{\sigma} \sqrt{l}-C_{\sigma}\right)^{2}, \quad B_{\sigma}=\sigma_{a 0} \sqrt{\pi} Y, \\
C_{\sigma}=\frac{\sqrt{2 \pi r}}{\Phi}\left(\sigma_{e r}+a_{\sigma r} \sqrt{f}\right) .
\end{gathered}
$$

Thus, based on the unified physical approach to describing the formation of the local plastic deformation zone during the fatigue fracture incubation and propagation periods, we obtained fatigue curve equations and dependences of the crack growth rate on $\Delta K$ and crack length that take into account the loading frequency and stress ratio. Physically, the equations for the crack growth rate are the constitutive equations of a material at the tip of a growing crack at the instant of time preceding the next onset of the crack growth.

The validity of the equations obtained was verified on a large volume of test data on nickel-, titanium-, and aluminum-based alloys and steels. Equations of the fatigue-crack growth curves (7) and (8) enable description of the fatigue-crack growth behavior, in particular at the stages of short crack development and stable or unstable crack growth, as well as conditions of the crack growth retardation up to the complete arrest.

The mathematical consequences of the equations are analytic dependences of the crack growth rate vs crack length, threshold stress-intensity factor $\Delta K_{t h}$ vs frequency $f$, crack growth rate $d l / d N$ vs frequency $f$ for $\Delta K=$ const, and cyclic crack-growth resistance characteristics $\Delta K_{t h}$ and $\Delta K_{f c}$ vs structural element size $d$.

1. V. V. Panasyuk and V. P. Sylovanyuk, "A computational model of fatigue fracture of materials," Fiz.-Khim. Mekh. Mater., No. 3, 44-54 (2003).

2. O. P. Ostash and V. V. Panasyuk, "Initiation of fatigue macrocracks in notched specimens," Strength Mater., 32, No. 5, 417-426 (2000). 
3. O. P. Ostash, V. V. Panasyuk, and E. M. Kostyk, "A unified model of initiation and growth of fatigue macrocracks. Pt. 2. Application of strain-based fracture mechanics parameters at the crack initiation stage," Fiz.-Khim. Mekh. Mater., No. 3, 5-14 (1998).

4. O. P. Ostash, V. V. Panasyuk, and E. M. Kostyk, "A unified model of initiation and growth of fatigue macrocracks. Pt. 3. Macrocrack growth stage," Fiz.-Khim. Mekh. Mater., No. 3, 55-66 (1999).

5. O. P. Ostash and I. M. Andreiko, "Cyclic crack growth resistance of high-strength cast irons at macrocrack nucleation and growth stages," Fiz.-Khim. Mekh. Mater., No. 1, 57-62 (2001).

6. V. P. Golub and A. V. Plashchinskaya, "Subcritical growth of fatigue cracks in thin plates with a stress concentrator," in: Proc. of the Int. Conf. on Life Assessment and Management for Structural Elements (Kiev, 2000), Vol. 1, Kiev (2000), pp. 93-99.

7. S. Kocanda, Zmeczeniowe Niszczenie Metali, Widawnictwo NaukowoTechniczne, Warzawa (1972).

8. X. Q. Shi, H. L. J. Pang, W. Zhou, and Z. P. Wang, "A modified energy-based low cycle fatigue model for eutectic solder alloy," Scr. Mater., 41, No. 3, 289-296 (1999).

9. F. V. Antunes, J. M. Ferreira, C. M. Branco, and J. Burne, "Influence of stress state on high-temperature fatigue crack growth in Inconel 718," Fatigue Fract. Eng. Mater. Struct., 24, No. 2, 127-135 (2001).

10. A. B. O. Soboyejo, S. Shademan, M. Foster, et al., "A multiparameter approach to the prediction of fatigue crack growth in metallic materials," Fatigue Fract. Eng. Mater. Struct., 24, No. 3, 225-241 (2001).

11. A. T. Yokobori and T. Isogai, "Fatigue crack growth and dislocation dynamics," J. Jap. Soc. Strength Fract. Mater., 34, No. 1, 1-10 (2000).

12. T. Yu. Yakovleva, Local Plastic Deformation and Fatigue of Metals [in Russian], Naukova Dumka, Kiev (2003).

13. A. V. Gur'ev and V. Ya. Mitin, "Features of development of nonuniform microscopic strains and fatigue damage accumulation in carbon steels," Strength Mater., 10, No. 11, 1263-1267 (1978).

14. C. Buque, W. Tirsher, and Ch. Blochwitz, "Rauschen in mechanische ermüdeten Nickeleinkristallen," Z. Metallik, 86, No. 10, 671-681 (1995).

15. T. Yu. Yakovleva and L. E. Matokhnyuk, "Estimation of degradation of a metallic material structure using the Fourier analysis method," in: Proc. of the Int. Conf. on Life Assessment and Management for Structural Elements (Kiev, 2000), Vol. 1, Kiev (2000), pp. 193-197.

16. T. Yu. Yakovleva, "Application of the methods of Fourier optics for quantitative analysis of the evolution of structural states of metals under conditions of cyclic loading," Strength Mater., 32, No. 2, 162-167 (2000).

17. M. D. Halliday, P. Poole, and P. Bowen, "New perspective on sleep band decohesion as fracture event during fatigue crack growth in both small and long cracks," Mater. Sci. Technol., 15, No. 4, 382-390 (1999). 
18. A. Sugeta, M. Jono, and Y. Uematsu, "Observation of fatigue crack growth behavior using an atomic force microscope," in: Proc. of the Seventh Int. Congress on Fatigue'99 (Beijing, P. R. China, 8-12 June, 1999), Vol. 4, Higher Education Press, Beijing (1999), pp. 2783-2788.

19. L. E. Matokhnyuk and T. Yu. Yakovleva, "Influence of loading frequency on the rules and mechanisms of fatigue crack growth in titanium alloys. Report 2," Strength Mater., 20, No. 1, 25-36 (1988).

20. T. Yu. Yakovleva and L. E. Matokhnyuk, "The influence of the cyclic loading rate on the plastic zone depth in VNS-25 alloy," Strength Mater., 34, No. 2, 150-152 (2002).

21. L. D. Landau and E. M. Lifshitz, Contimuum Mechanics [in Russian], Gostekhizdat, Moscow-Leningrad (1953).

22. C. Zener, Elasticity and Inelasticity of Metals, Chicago (1948).

23. L. M. Kachanov, Fundamentals of Fracture Mechanics [in Russian], Nauka, GRFML, Moscow (1974).

24. G. Pluvinage, "Notch fracture mechanics," Fiz.-Khim. Mekh. Mater., No. 6, 7-20 (1998). 